\title{
Sequential Geometric Approach for Device-Free Localization with Outlier Link Rejection
}

\author{
Wendong Xiao and Biao Song \\ School of Automation \& Electrical Engineering, University of Science and Technology Beijing, Beijing 100083, China \\ Correspondence should be addressed to Wendong Xiao; wendongxiao68@163.com
}

Received 3 October 2014; Revised 16 January 2015; Accepted 28 January 2015

Academic Editor: Mehmet Onder Efe

Copyright (C) 2015 W. Xiao and B. Song. This is an open access article distributed under the Creative Commons Attribution License, which permits unrestricted use, distribution, and reproduction in any medium, provided the original work is properly cited.

\begin{abstract}
Device-free localization (DFL) is an emerging technique for estimating the location of the target that does not attach any electronic equipment. Wireless devices are needed to perform as transmitters or receivers. The location of the target is estimated by detecting the changes of the received signal strength (RSS) measurements of the wireless links formed by wireless transmitters and receivers. Due to the uncertainty of the wireless channel, certain links may be polluted seriously, resulting in error detection. In this paper, we propose a novel sequential geometric approach with outlier link rejection (SGOLR) for DFL. It consists of three sequential strategies, including (1) affected link identification by differential RSS detection; (2) outlier link rejection via clustering algorithm by intersection of link (IoL) calculation from the affected links; and (3) density based IoL selection and target location estimation from the remained IoLs. Experimental results show that SGOLR is robust to the fluctuation of the wireless signals with superior localization accuracy compared with the existing Radio Tomographic Imaging (RTI) approach.
\end{abstract}

\section{Introduction}

Wireless localization has many applications with promising commercial value; correspondingly, various techniques have been developed, for example, based on GPS [1], ultrasound $[2,3]$, Infrared [4], and radio-frequency (RF) [5-7]. In such techniques, the target to be localized will be equipped with electronic device, which is inconvenient and impractical in many scenarios (e.g., in medical healthcare, security safeguard, and emergency rescue).

Recently device-free localization (DFL) is receiving tremendous interests for localization of target without attaching any device. In DFL system, a number of signal transmitters and signal receivers are deployed. It is found that, when the target enters the monitoring area, the RSS measurements of certain links between the signal transmitters and signal receivers will change significantly [8-12], and the signal variations will be used to estimate the target location.

DFL is remaining as a challenging problem, mainly due to the uncertain and dynamic wireless propagation environment suffering from non-line-of-sight, multipath, and fading phenomena. Some links, even without the presence of the targets, may be found to be with large RSS signal changes and cause error detection. If such links are used in the location estimation, the accuracy of the localization algorithm will be degraded significantly. However, most existing DFL approaches, such as the Radio Tomographic Imaging (RTI) approach [9-11] and the geometrical approach $[13,14]$, do not consider such links explicitly. Although [15] considers the outlier link rejection, only the links without intersections with all the other links are rejected and may still keep wrong links in many situations.

In this paper, we will propose a novel sequential geometric with outlier link rejection (SGOLR) for DFL by a clustering [16] based outlier link rejection strategy via the intersection calculation of the affected links, as well as an intersection selection scheme.

The paper is organized as follows. The related work is introduced in Section 2; signal model is given in Section 3; the details of the proposed approach are addressed in Section 4; the experimental results are reported in Section 5; finally, conclusions and the future work are given in Section 6 . 


\section{Related Work}

A nubmer of DFL approaches have been proposed, including fingerprinting approaches based on the differential RSS measurements, geometric approaches, RTI approaches, and compressive sensing approaches.

El-Kafrawy et al. investigate the impacts of the human motion on the variances of the RSS measurements of wireless links [8]. Youssef et al. propose the fingerprinting approach for DFL $[17,18]$. A radio map is built at the offline phase by recording the differential RSS measurements of links when the target is located at reference points with known locations. In the online phase, the location of the target is estimated by comparing the measured differential RSS signals of the links with the offline radio map. Although the performance of this approach is acceptable, a tedious calibration procedure is required. It is also challenging to build the radio map efficiently.

As a typical geometric approach, Zhang et al. propose a signal dynamics model for tracking transceiver-free targets and three tracking algorithms by a number of geometric schemes such as the midpoint, the intersection, and the bestcover calculations $[13,14]$.

Wilson and Patwari propose the RTI approach based on the link differential RSS measurements between the measured RSS measurements of links and the RSS measurements of links without the target via the reconstruction of the tomography image for the locations of the target [9-11]. The ill-posed inverse problem is derived and solved by the regularization method. Kaltiokallio et al. present an online recalibration method to adapt the changes in the radio environment [19].

Wang et al. propose the compressive sensing approaches to tackle the ill-posed inverse problem in signal reconstruction from the the RSS measurements [20-22].

The above-mentioned approaches do not consider the negative effects of the outlier links for the localization, and the localization accuracy is limited.

\section{Signal Model}

Suppose there are $N$ wireless nodes as transmitters or receivers for detecting the target. If wireless node $i$ and wireless node $j$ can communicate with each other directly, then we denote the link by $L_{n}(i, j)$ and $L_{n}$ for simplicity, with $n$ being the ID of the link. We assume that the total number of links in the system is $P$.

Let $X_{i}\left(x_{i}, y_{i}\right)$ denote the location of the wireless node $i$ in the Cartesian coordinates and $\mathrm{RSS}_{n}^{0}$ and $\mathrm{RSS}^{0}=\left\{\mathrm{RSS}_{1}^{0}\right.$, $\left.\operatorname{RSS}_{2}^{0}, \ldots, \operatorname{RSS}_{P}^{0}\right\}$ denote the RSS measurements of link $n$ and the RSS measurements of all links when the monitoring area is vacant. Also, let $\mathrm{RSS}_{n}^{t}$ and RSS $\mathrm{RS}^{t}=\left\{\mathrm{RSS}_{1}^{t}, \mathrm{RSS}_{2}^{t}, \ldots, \mathrm{RSS}_{P}^{t}\right\}$ denote the RSS measurements of link $n$ and the RSS measurements of all links at time $t$.

Mathematically, when the monitoring area is vacant, $\operatorname{RSS}_{n}^{0}$ can be calculated by

$$
\mathrm{RSS}_{n}^{0}=P_{n}-D_{n}-F_{n}(0)-v_{n}(0),
$$

where

(i) $P_{n}$ is the transmitting power of the transmitter $(\mathrm{dB})$,

(ii) $D_{n}$ is the static losses due to device inconsistencies, distance, antenna patterns, and so forth in $\mathrm{dB}$,

(iii) $F_{n}(0)$ is the random fading loss when the monitoring area is vacant and occurs from constructive and destructive interference of narrowband signals in multipath environments in $\mathrm{dB}$,

(iv) $v_{n}(0)$ is the measurement noise when the monitoring area is vacant.

$\mathrm{RSS}_{n}^{t}$ at time $t$ when the target is present can be calculated as

$$
\mathrm{RSS}_{n}^{t}=P_{n}-D_{n}-S_{n}(t)-F_{n}(t)-v_{n}(t),
$$

where, different from (1),

(i) $S_{n}(t)$ is the shadowing loss at time $t$ due to the target that attenuates the signal in $\mathrm{dB}$,

(ii) $F_{n}(t)$ is the fading loss at time $t$ that occurs from constructive and destructive interference of narrowband signals in multipath environments in $\mathrm{dB}$,

(iii) $v_{n}(t)$ is measurement noise at time $t$.

The difference between the RSS of links with the target at time $t$ and without the target in the monitoring area is

$$
\begin{aligned}
\Delta \mathrm{RSS}_{n}^{t} & =\mathrm{RSS}_{n}^{t}-\mathrm{RSS}_{n}^{0} \\
& =S_{n}(t)+F_{n}(t)-F_{n}(0)+v_{n}(t)-v_{n}(0),
\end{aligned}
$$

where the noise consists of the fading and the measurement noises and is denoted as

$$
w_{n}(t)=F_{n}(t)-F_{n}(0)+v_{n}(t)-v_{n}(0) .
$$

Then, we have

$$
\Delta \mathrm{RSS}_{n}^{t}=S_{n}(t)+w_{n}(t) .
$$

From (5), the main contribution of the $\Delta \mathrm{RSS}_{n}^{t}$ of link $n$ pertains to the presence of the target and the noise. We call the links with large differential RSS as the affected links. Due to the uncertain wireless propagation environment, some links associated with large noise but are far away from the target may be incorrectly identified as the affected links and degrade the accuracy of the localization algorithms significantly if they are considered in location estimation. Thus, such links are considered as the outlier links in this paper and will be rejected in the localization approach.

\section{The Details of the Proposed Approach}

SGOLR consists of three key strategies sequentially implemented, including the affected link identification by differential RSS detection, clustering based outlier link rejection, and density based intersection of link (IoL) selection and target location estimation. The details of SGOLR will be given in this section. 


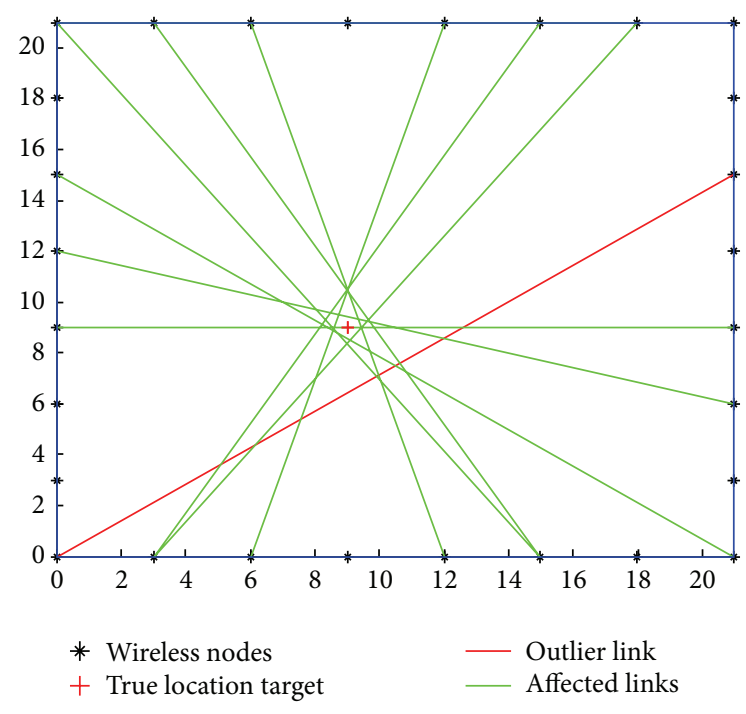

FIGURE 1: The affected links and the outlier link.

4.1. Affected Link Identification. Finding the affected links is essential for the localization algorithms. In the offline phase, the radio map can be built by collecting the RSS measurements of links; that is, $\mathrm{RSS}^{0}=\left\{\mathrm{RSS}_{1}^{0}, \mathrm{RSS}_{2}^{0}, \ldots, \mathrm{RSS}_{P}^{0}\right\}$. At time $t$, RSS ${ }^{t}$ can be obtained and the differential RSSs of links $\left(\Delta \mathrm{RSS}^{t}=\left\{\Delta \mathrm{RSS}_{1}^{t}, \Delta \mathrm{RSS}_{2}^{t}, \ldots, \Delta \mathrm{RSS}_{P}^{t}\right\}\right)$ can be calculated. A negative scalar threshold $\gamma$ is used to identify whether a link is an affected link and whether the target is present in the monitoring area [23]. Normally, if the link $n$ is affected by the target, $\Delta \mathrm{RSS}_{n}^{t}$ will be less than a threshold $\gamma[24,25]$. Therefore, we define the state of the link $n$ as

$$
s_{n}^{t}= \begin{cases}1, & \text { if } \Delta \mathrm{RSS}_{n}^{t} \leq \gamma \\ 0, & \text { if } \Delta \mathrm{RSS}_{n}^{t} \geq \gamma .\end{cases}
$$

If $s_{n}^{t}=1$, then link $n$ will be considered as the affected link. The states of all links $s^{t}=\left\{s_{1}^{t}, s_{2}^{t}, \ldots, s_{P}^{t}\right\}$ can be obtained according to (6).

4.2. Outlier Link Rejection. As shown in Figure 1, due to the noise of the wireless link, a link may be identified as an affected link incorrectly when its noise is serious. The affected links that can detect the target will be around the target and near from each other, and we define an outlier link as an affected link that is separated and far away from those affected links around the target.

In SGOLR, the outlier links are identified and rejected by clustering the identified affected links via IoL calculation. In this paper, we only consider the localization problem for the single target.

The geometrical relationship of links is used to detect whether the outlier links are present inside the affected links.

The affected links can be obtained from the states of links $s^{t}=\left\{s_{1}^{t}, s_{2}^{t}, \ldots, s_{P}^{t}\right\}$, and we assume that the number of the affected links is $m$. The number of intersections is less than or equal to $m(m-1) / 2$. We assume that the number of intersections is $\mathscr{M}$. Because the locations of wireless nodes are known, the locations of intersections can be calculated. Here, we use $X_{i, j}\left(x_{i, j}, y_{i, j}\right)$ to denote the location of the intersection of link $i$ and link $j$.

Definition 1. The center of the intersections $\left(x_{c}, y_{c}\right)$ is the average coordinate of the intersections of the affected links

$$
\begin{aligned}
& x_{c}=\frac{\sum_{i=1}^{P} \sum_{j=1}^{P} s_{i}^{t} s_{j}^{t} x_{i, j}}{\mathscr{M}}, \\
& y_{c}=\frac{\sum_{i=1}^{P} \sum_{j=1}^{P} s_{i}^{t} s_{j}^{t} y_{i, j}}{\mathscr{M}} .
\end{aligned}
$$

The geometrical relationship of links is reflected in the distance values $\operatorname{dis}^{t}=\left\{\operatorname{dis}_{1}^{t}, \operatorname{dis}_{2}^{t}, \ldots, \operatorname{dis}_{\mathcal{M}}^{t}\right\}$ where $\operatorname{dis}_{n}^{t}$ denotes the distance between the affected link $n$ and the center of the intersections $\left(x_{c}, y_{c}\right)$. The distance between the outlier link and the center of the intersections is always greater than the distance between the affected links and the center of the intersections. A threshold of distance $(\delta)$ is selected empirically to detect the outlier links

$$
\delta=\frac{2 \sum_{i=1}^{m} \operatorname{dis}_{i}}{m} .
$$

The outlier state of link $n(1 \leq n \leq m)$ can be determined by

$$
e_{n}^{t}= \begin{cases}1, & \text { if } \operatorname{dis}_{n}^{t}>\delta \\ 0, & \text { otherwise }\end{cases}
$$

Then, the outlier state of the affected links can be calculated as

$$
o_{t}= \begin{cases}1, & \text { if } \sum_{i}^{m} e_{i}^{t} \geq 1 \\ 0, & \text { otherwise. }\end{cases}
$$

If $o_{t}=0$, then we consider that no outlier link exists in the affected links. Whereas if $o_{t}=1$, there are outlier links that will be rejected from the affected links, and the following clustering operation will be performed.

Firstly, the distances associated with the links dis ${ }^{t}=\left\{\right.$ dis $_{1}^{t}$, $\left.\operatorname{dis}_{2}^{t}, \ldots, \operatorname{dis}_{m}^{t}\right\}$ are considered as a data set $\left\{x_{1}, x_{2}, \ldots, x_{m}\right\}$ and clustered by the $K$-means algorithm [16]. Two clusters are generated for the affected links and outlier links, respectively, and two corresponding centroids are calculated, one with the distance less than $\delta$ and the other with the distance larger than $\delta$. Then, each data in the data set is clustered to the nearest centroid, and the centroids will be updated by recalculating the average data in each cluster [26]. With the new centroids, a new loop is formed and each data is associated with the nearest new centroid. Finally, the loop stops until the centroids are not changed any more. This approach aims at minimizing an objective function [16] described as

$$
J=\sum_{j=1}^{2} \sum_{i=1}^{m}\left\|x_{i}^{j}-c_{j}\right\|^{2},
$$


(1) Get the $\Delta \mathrm{RSS}^{t}$ according to (5),

(2) Get the state of links according to (6),

(3) If there are affected links,

(4) Calculate the coordinates of the intersections,

(5) Calculate the center of the intersections $\left(x_{c}, y_{c}\right)$ according to (7),

(6) Get the threshold of distance $(\delta)$ according to (8),

(7) Estimate the outlier state of links according to (10),

(8) If there are outlier links (outlier state is 1),

(9) Cluster the affected links using $K$-means algorithm based on the distance values $\operatorname{dis}^{t}=\left\{\operatorname{dis}_{1}^{t}, \operatorname{dis}_{2}^{t}, \ldots, \operatorname{dis}_{m}^{t}\right\}$.

(10) The links in the cluster with the larger centroid coordinate is the outlier links,

(11) Eliminate the outlier links.

(12) End

(13) End

Algorithm 1: Detect and reject outlier links.

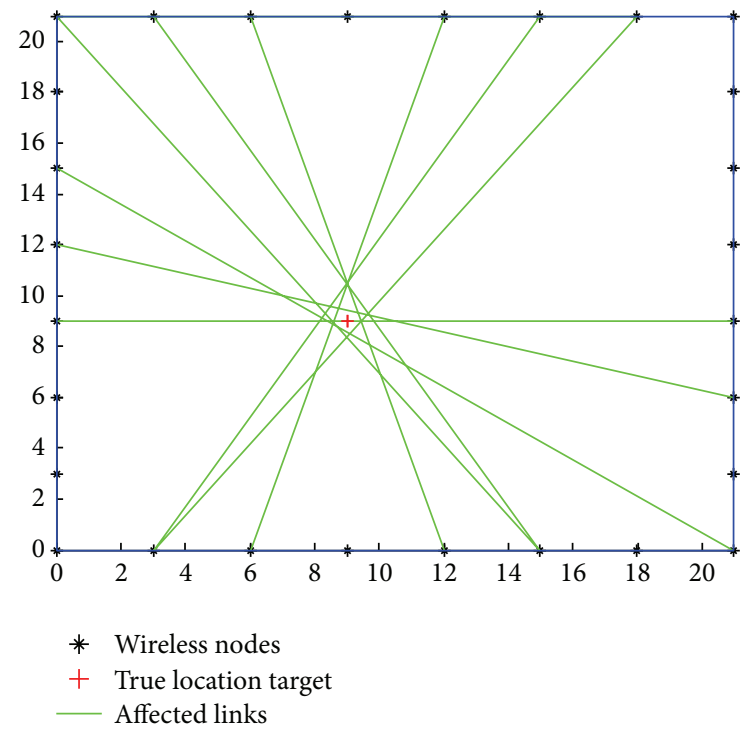

FIGURE 2: Affected links with outlier link rejection.

where $x_{i}^{j}$ is the associated data in the data set and $c_{j}$ is the centroid. There are only two centroids, one for the affected links and the other for the outlier links. Because the distance between the center of the intersections and an outlier link is larger than the distance between the center of the intersections and the affected link, the centroid with the larger coordinate should belong to the outlier links. Finally, the cluster with the larger centroid coordinate is associated with the set of the outlier links.

The process that detects and reject outlier links can be summarized in Algorithm 1.

4.3. IoL Selection and Target Location Estimation. As shown in Figure 2, the true target location is associated with the affected links. The target location can be estimated from the affected links and the intersections of the affected links. Although the outlier links are rejected, the noise may still degrade the accuracy of the localization algorithm.

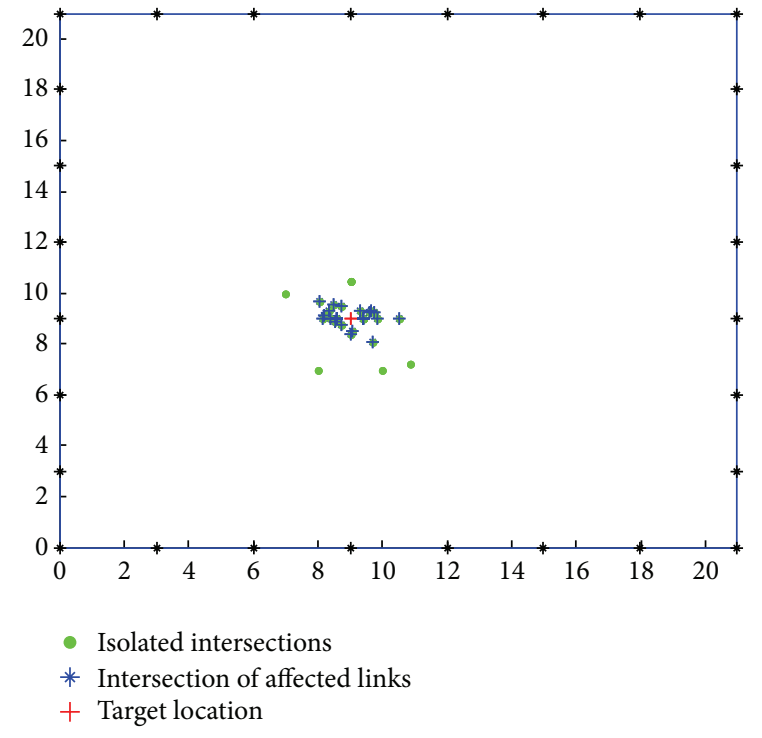

FIGURE 3: IoLs with the isolated intersections.

As shown in Figure 3, some intersections are isolated and have influence on the localization algorithm. In order to increase the accuracy of the localization algorithm, the isolated intersections should be eliminated. For this purpose, the density of IoLs is defined.

Definition 2. The density of IoL refers to the number of intersections with distances from it less than a given radius.

As shown in Figure 4, the density of IoL $\left(x_{i}, y_{i}\right)$ is the number of intersections in the circle with the center $\left(x_{i}, y_{i}\right)$ and the radius $R$. In this paper, we study the scenario with the rectangle monitoring area and the equal distance between the adjacent wireless devices.

All the isolated intersections with density value 1 will be eliminated. Then, the coordinate of the intersections without the isolated intersections can be obtained and their averaged 
(1) Use the Algorithm 1 to detect and eliminate outlier links,

(2) Get the coordinates of the intersections of the effect links,

(3) Use the density of IoL to detect and eliminate the isolated intersections.

(4) Estimate the coordinate of the target $\left(x_{\text {target }}, y_{\text {target }}\right)$ according to (12).

Algorithm 2: The process of SGOLR.

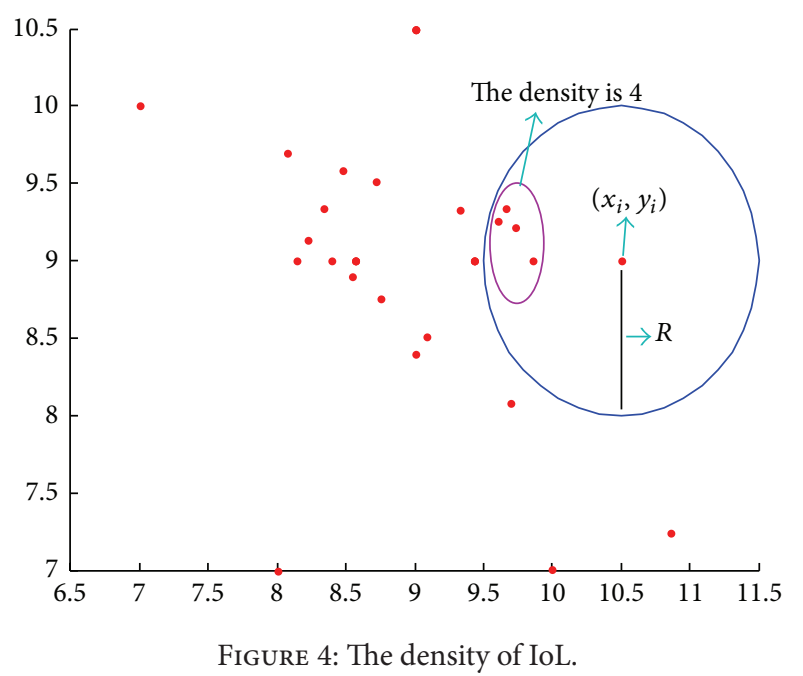

coordinate $\left(x_{\text {target }}, y_{\text {target }}\right)$ will be calculated as the location estimation of the target; that is,

$$
\begin{aligned}
& x_{\text {target }}=\frac{\sum_{i=1}^{u} x_{i}}{u}, \\
& y_{\text {target }}=\frac{\sum_{i=1}^{u} y_{i}}{u},
\end{aligned}
$$

where $u$ is the number of the intersections without the isolated intersections.

The process of SGOLR is summarized in Algorithm 2.

Now, we give the complexity analysis for SGOLR. Assume that the number of loops in the $K$-means algorithm is $q$ and the computational load for Algorithm 1 is $O(q m)$. The computational load for SGOLR (Algorithm 2) is $u^{2}+q m$, as $\mathscr{M}(\mathscr{M}-1) / 2 \geq u>\mathscr{M}$; we can know the complexity for SGOLR is $O\left(u^{2}\right)$.

\section{Experimental Evaluation}

5.1. Experimental Setup. To evaluate the performance of SGOLR, we performed the experiments using the experimental data that can be acquired [27] from the SPAN Lab of the University of Utah. There are 28 wireless devices, and the total number of links is 378 . The RSS of each link is an averaged value of the RSS measurements from bidirectional transmissions. Each wireless device is placed three feet apart along the perimeter of a $21 * 21$ feet square, surrounding a total area of 441 square feet, and is placed on a stand at three feet off the ground. In the experiment, the target is the person

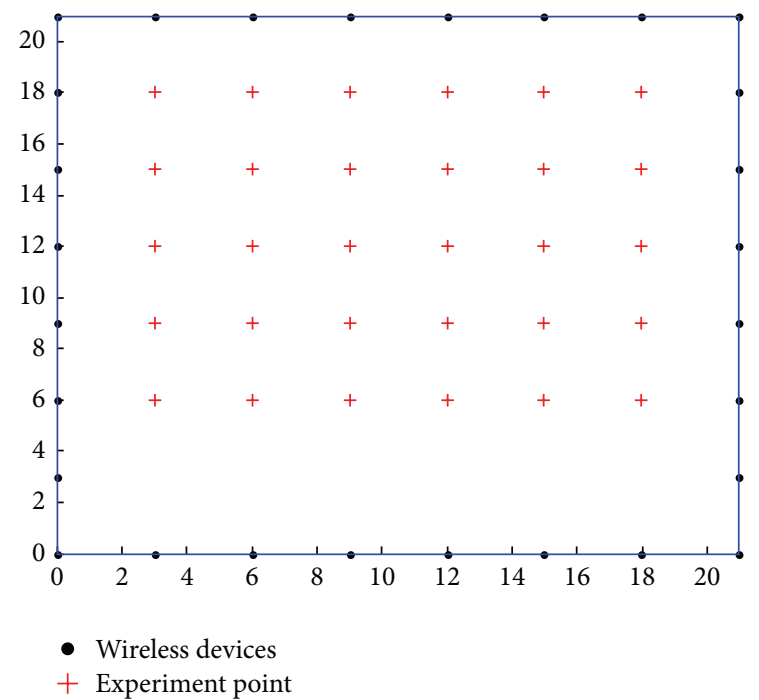

Figure 5: The experimental setup.

TABLE 1: Parameters of RTI.

\begin{tabular}{lcc}
\hline Parameter & Value & Description \\
\hline$\Delta p$ & 0.5 & Voxel width (feet) \\
$\varepsilon$ & 0.01 & Width of weighting ellipse (feet) \\
$\alpha$ & 5 & Regularization parameter \\
\hline
\end{tabular}

with the height $1.85 \mathrm{~m}$ and the weight $88 \mathrm{~kg}$. As shown in Figure 5, 30 positions are selected for testing and comparing SGOLR with RTI [9].

In RTI, the monitored area is divided into voxels; aggregately, all voxels contribute to the RSS change of each transmitter-receiver link in the network. The weights of all voxels are computed according to their impacts on all the links, and the voxel with the minimum weight is considered as the location estimation of the target. The RTI reconstruction for obtaining the weights of the voxels uses $\mathrm{H} 1$ regularization with the parameters listed in Table 1.

The parameters of SGOLR are listed in Table 2. As the analytical relationship between the parameters and the system performance of SGOLR is not clear, we use the trial and error method to determine the parameters.

The localization errors against different threshold $\gamma$ are shown in Figures 6(a) and 6(b). If $\gamma \leq-7.5 \mathrm{~dB}$, there may be not enough affected links for localization. The accuracy of localization for some positions is destroyed, and the average error increases. Meanwhile, from Figure 6(a), if $\gamma \geq-3 \mathrm{~dB}$, big errors are found for some positions, as there are too many 


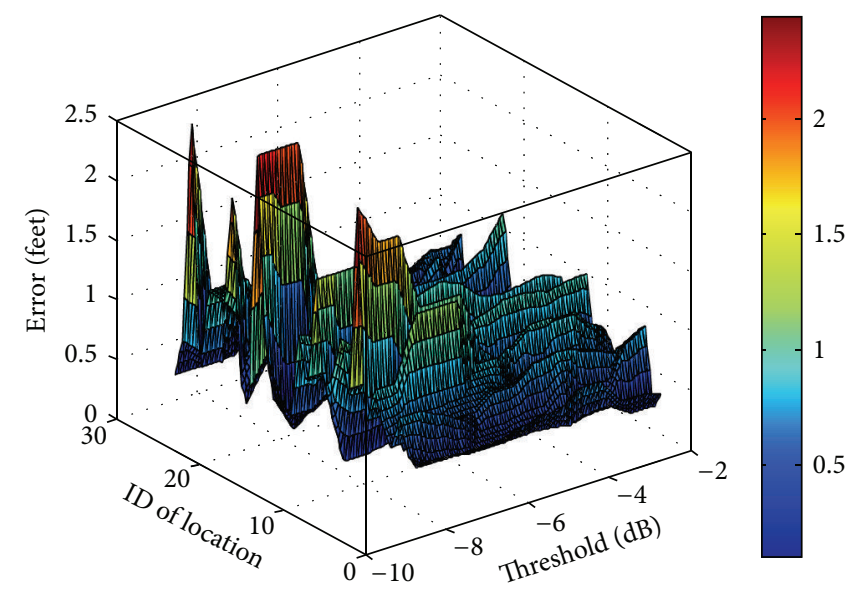

(a) The errors when $\gamma$ changes

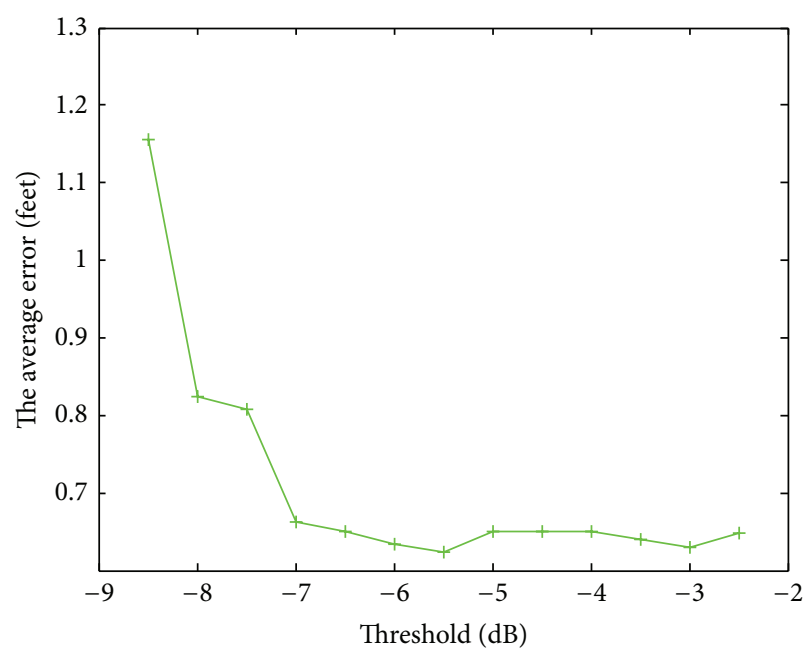

(b) Average errors for different $\gamma$

FIGURE 6: Localization errors against different threshold $\gamma$.

TABLE 2: Parameters of SGOLR.

\begin{tabular}{lcl}
\hline Parameter & Value & Description \\
\hline$\gamma$ & -7 & $\begin{array}{l}\text { The threshold used for affected link } \\
\text { detection (dB) } \\
R\end{array}$ \\
1 & $\begin{array}{l}\text { The radius for density calculation of IoL } \\
\text { (feet) }\end{array}$ \\
\hline
\end{tabular}

affected links, including some outlier links that can not be rejected.

The localization errors against different radius $R$ for density of IoL are shown in Figures 7(a) and 7(b). If $R \leq 0.7$, some unisolated intersections are eliminated. The accuracy of localization for some positions is destroyed, and the average error is large.

5.2. Performance Comparison. To evaluate the performance of SGOLR, we compare it with the localization approach based on the intersections of the links without outlier link rejection (IoLwOLR), the localization approach based on the intersections of the affected links with outlier link rejection and without intersection selection (IoLwIS), and RTI [9]. The Euler distance between the "true" location of the target and the estimated location of the target is used as the localization error.

IoLwOLR obtains the affected links without outlier link rejection and calculates the intersections of the affected links. The target location is calculated by averaging the coordinates of the intersections.

IoLwIS obtains the affected links, rejects the outlier links, and calculates the intersections of the affected links. The target location is obtained by averaging the coordinates of the intersections.

The comparison results are shown in Figure 8 and Table 3. It is obvious that IoLwIS has better performance than IoLwOLR. The mean localization error and the worst localization errors of IoLwIS are reduced by $44 \%$ and $54 \%$, respectively, from those of IoLwOLR, due to the outlier link rejection strategy.

By comparing IoLwIS and SGOLR, it is shown that the isolated intersections are influential for the accuracy. The worst accuracy, the best accuracy, and the mean accuracy are polluted by the isolated intersections. It demonstrates that eliminating the isolated intersections is good for improving the localization performance.

It is obvious that the SGOLR has better performance than the RTI. The mean, variance, worst, and best of the localization error are all significantly improved.

Outlier links are not eliminated in IoLwOLR and RTI and are eliminated in IoLwIS and SGOLR. RTI uses all links to reconstruct the tomography image of the location of the target, and IoLwOLR uses the affected links which may contain the outlier links. From Figure 8 and Table 3, it is obvious that the outlier links are disgusting for the accuracy of localization algorithm and the outlier link rejection can significantly reduce the localization error. The robustness and reliability of the DFL systems are destroyed by the outlier links. Eliminating the outlier links can also improve the variances, the worst errors, and the best localization errors significantly, demonstrating that the DFL with outlier link rejection is more robust and reliable.

The RSS measurements of links are measured under the complex dynamic environment, and the intersections of links are mixed with the noise. In this paper, eliminating the isolated intersections is used as the second time filter. The experimental results between IoLwIS and SGOLR in Figure 8 and Table 3 verify that it is necessary to use IoL selection for improving the localization accuracy.

\section{Conclusions}

This paper proposes a novel sequential geometric approach SGOLR for DFL. To decrease the disgusting impacts of 


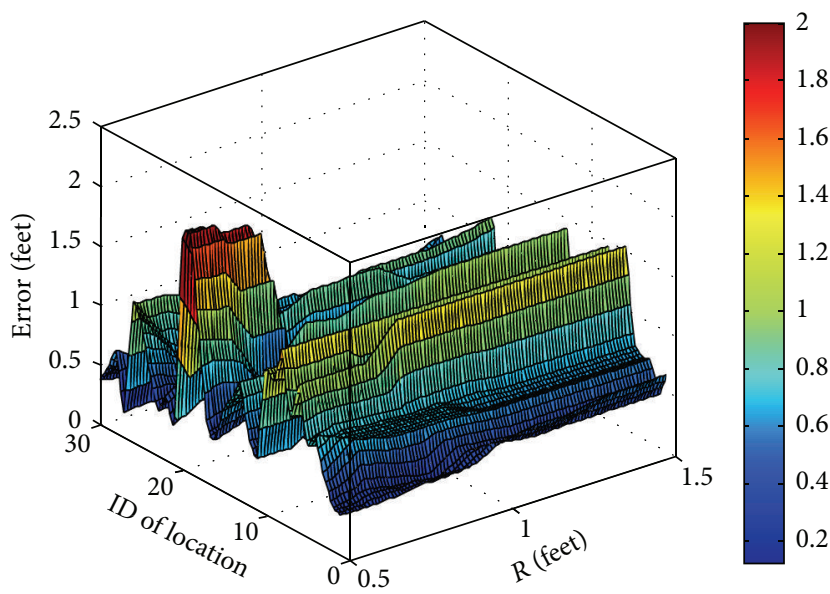

(a) The errors when $R$ changes

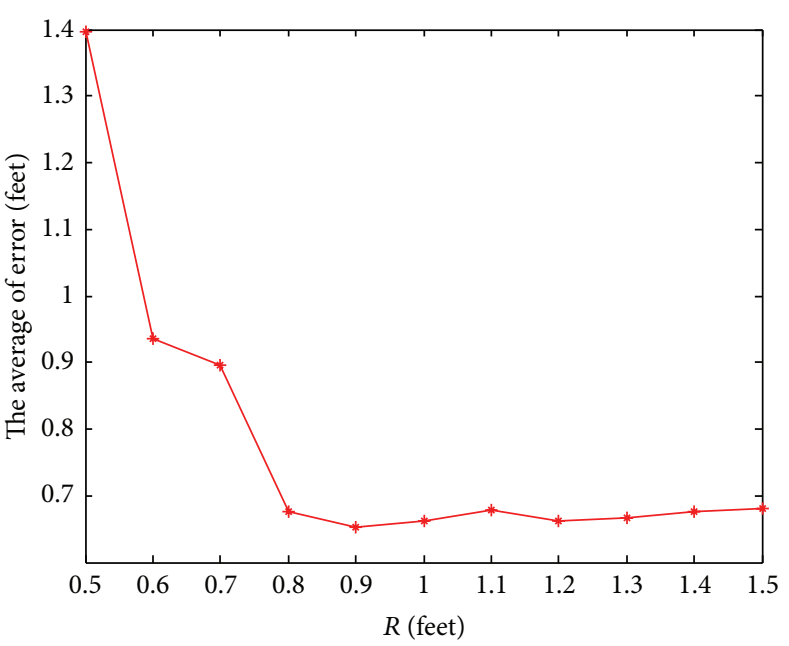

(b) Average errors for different $R$

FIGURE 7: Localization errors against different radius $R$.

TABLE 3: Performance comparison of 4 different approaches.

\begin{tabular}{lcccc}
\hline Algorithm & Error mean (feet) & Error variance & Error worst (feet) & Error best (feet) \\
\hline SGOLR & 0.6621 & 0.1336 & 1.5334 & 0.0430 \\
IoLwOLR & 1.2517 & 0.5310 & 3.3439 & 0.1549 \\
IoLwOIS & 0.7254 & 0.1231 & 1.5334 & 0.1549 \\
RTI & 0.8244 & 0.4262 & 3.8891 & 0.3536 \\
\hline
\end{tabular}

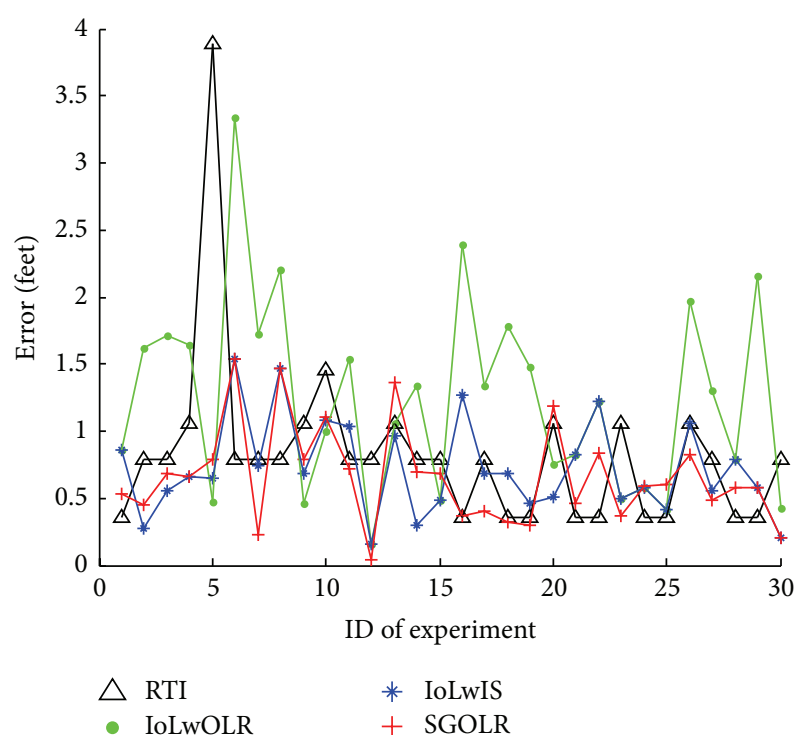

FIGURE 8: Performance comparison.

the large noises of the wireless links, outlier link detection and rejection are suggested by considering the geometrical relationship of links and appeal to the clustering algorithm. The density of IoL is used to eliminate the isolated intersections. Experimental results show the superior localization performance of SGOLR. As a future work, more solid mathematical study for SGOLR (e.g., in the optimization framework) and
DFL for multiple targets are two important problems for further studies.

\section{Conflict of Interests}

The authors declare that there is no conflict of interests regarding the publication of this paper.

\section{References}

[1] P. Enge and P. Misra, "Special issue on global positioning system," Proceedings of the IEEE, vol. 87, no. 1, pp. 3-15, 1999.

[2] N. B. Priyantha, A. Chakraborty, and H. Balakrishnan, "The Cricket location-support system," in Proceedings of the 6th Annual International Conference on Mobile Computing and Networking (MOBICOM '00), pp. 32-43, August 2000.

[3] W. Xiao, J. K. Wu, L. Shue, Y. Li, and L. Xie, "A prototype ultrasonic sensor network for tracking of moving targets," in Proceedings of the 1st IEEE Conference on Industrial Electronics and Applications (ICIEA '06), pp. 1511-1516, May 2006.

[4] R. Want, A. Hopper, V. Falcao, and J. Gibbons, "The Active badge location system," ACM Transactions on Information Systems, vol. 10, no. 1, pp. 91-102, 1992.

[5] W. Meng, W. Xiao, W. Ni, and L. Xie, "Secure and robust Wi-Fi fingerprinting indoor localization," in Proceedings of the International Conference on Indoor Positioning and Indoor Navigation (IPIN '11), pp. 1-7, IEEE, Guimarães, Portugal, September 2011.

[6] W. Xiao, P. Liu, W. S. Soh, and G. Huang, "Large scale wireless indoor localization by clustering and extreme learning 
machine," in Proceedings of the 15th International Conference on Information Fusion (FUSION '12), pp. 1609-1614, Singapore, July 2012.

[7] P. Bahl and V. N. Padmanabhan, "RADAR: an in-building RFbased user location and tracking system," in Proceedings of the 19th Annual Joint Conference of the IEEE Computer and Communications Societies (INFOCOM '00), vol. 2, pp. 775-784, March 2000.

[8] K. El-Kafrawy, M. Youssef, and A. El-Keyi, "Impact of the human motion on the variance of the received signal strength of wireless links," in Proceedings of the IEEE 22nd International Symposium on Personal, Indoor and Mobile Radio Communications (PIMRC '11), pp. 1208-1212, September 2011.

[9] J. Wilson and N. Patwari, "Radio tomographic imaging with wireless networks," IEEE Transactions on Mobile Computing, vol. 9, no. 5, pp. 621-632, 2010.

[10] J. Wilson and N. Patwari, "See-through walls: motion tracking using variance-based radio tomography networks," IEEE Transactions on Mobile Computing, vol. 10, no. 5, pp. 612-621, 2011.

[11] J. Wilson and N. Patwari, "A fade-level skew-laplace signal strength model for device-free localization with wireless networks," IEEE Transactions on Mobile Computing, vol. 11, no. 6, pp. 947-958, 2012.

[12] Y. Zhao, N. Patwari, J. M. Phillips, and S. Venkatasubramanian, "Radio tomographic imaging and tracking of stationary and moving people via kernel distance," in Proceedings of the 12th International Conference on Information Processing in Sensor Networks(IPSN '13), pp. 229-240, ACM, April 2013.

[13] D. Zhang, J. Ma, Q. Chen, and L. M. Ni, "An RF-based system for tracking transceiver-free objects," in Proceedings of the 5th Annual IEEE International Conference on Pervasive Computing and Communications (PerCom '07), pp. 135-144, March 2007.

[14] D. Zhang, K. Lu, R. Mao et al., "Fine-grained localization for multiple transceiver-free objects by using RF-based technologies," IEEE Transactions on Parallel and Distributed Systems, vol. 25, no. 6, pp. 1464-1475, 2014.

[15] J. Wang, Q. H. Gao, Y. Yu, P. Cheng, L. Wu, and H. Wang, "Robust device-free wireless localization based on differential RSS measurements," IEEE Transactions on Industrial Electronics, vol. 60, no. 12, pp. 5943-5952, 2013.

[16] J. M. Queen, "Some methods for classification and analysis of multivariate observations," in Proceedings of the Fifth Berkeley Symposium on Mathematical Statistics and Probability, vol. 1, pp. 281-297, University of California Press, Berkeley, Calif, USA, 1967.

[17] I. Sabek and M. Youssef, "Multi-entity device-free WLAN localization," in Proceedings of the IEEE Global Communications Conference (GLOBECOM '12), pp. 2018-2023, Anaheim, Calif, USA, December 2012.

[18] M. Seifeldin, A. Saeed, A. E. Kosba, A. El-Keyi, and M. Youssef, "Nuzzer: a large-scale device-free passive localization system for wireless environments," IEEE Transactions on Mobile Computing, vol. 12, no. 7, pp. 1321-1334, 2013.

[19] O. Kaltiokallio, M. Bocca, and N. Patwari, "Follow @grandma: long-term device-free localization for residential monitoring," in Proceedings of the IEEE 37th Conference on Local Computer Networks Workshops (LCN '12), pp. 991-998, Clearwater, Fla, USA, October 2012.

[20] J. Wang, Q. Gao, X. Zhang, and H. Wang, "Device-free localisation with wireless networks based on compressive sensing," IET Communications, vol. 6, no. 15, pp. 2395-2403, 2012.
[21] J. Wang, Q. H. Gao, H. Y. Wang, Y. Yu, and M. L. Jin, “Timeof-flight-based radio tomography for device free localization," IEEE Transactions on Wireless Communications, vol. 12, no. 5, pp. 2355-2365, 2013.

[22] J. Wang, D. Y. Fang, X. J. Chen, Z. Yang, T. Z. Xing, and L. Cai, "LCS: compressive sensing based device-free localization for multiple targets in sensor networks," in Proceedings of the 32nd IEEE Conference on Computer Communications (INFOCOM '13), pp. 145-149, April 2013.

[23] A. Saeed, A. E. Kosba, and M. Youssef, "Ichnaea: a low-overhead robust WLAN device-free passive localization system," IEEE Journal on Selected Topics in Signal Processing, vol. 8, no. 1, pp. 5-15, 2014.

[24] X. Y. Zheng, J. Yang, Y. Y. Chen, and Y. Gan, "Adaptive devicefree passive localization coping with dynamic target speed," in Proceedings of the 32nd IEEE Conference on Computer Communications (INFOCOM '13), pp. 485-489, April 2013.

[25] Y. Guo, K. Huang, N. Y. Jiang, X. M. Guo, and G. L. Wang, "An exponential-Rayleigh signal strength model for device-free localization and tracking with wireless networks," in Proceedings of the 4th International Conference on Intelligent Control and Information Processing (ICICIP '13), pp. 108-113, Beijing, China, June 2013.

[26] S. Guha, R. Rastogi, and K. Shim, "CURE: an efficient clustering algorithm for large databases," in Proceedings of the ACM SIGMOD International Conference on Management of Data (SIGMOD '98), pp. 73-84, June 1998.

[27] http://span.ece.utah.edu/rti-data-set. 


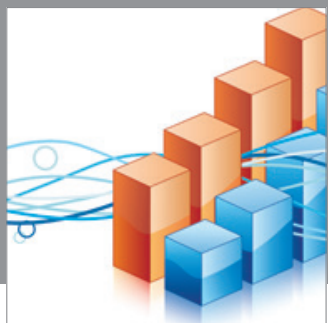

Advances in

Operations Research

mansans

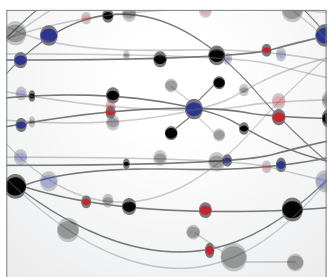

The Scientific World Journal
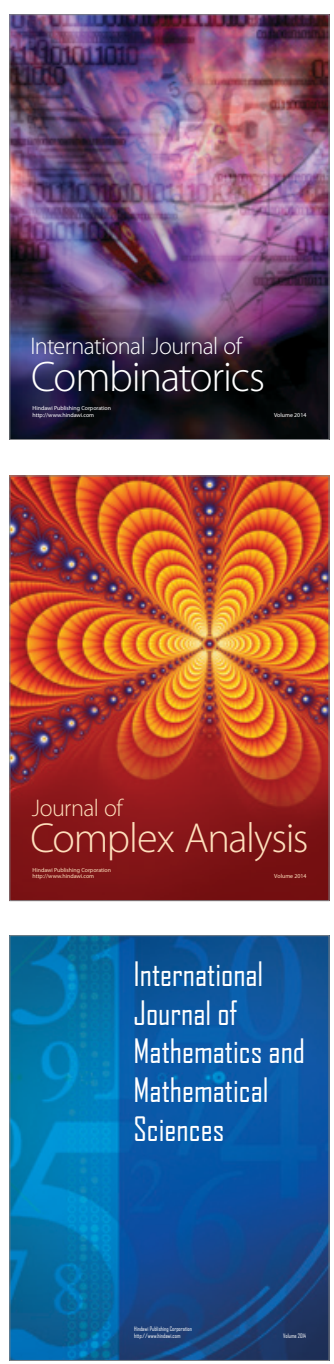
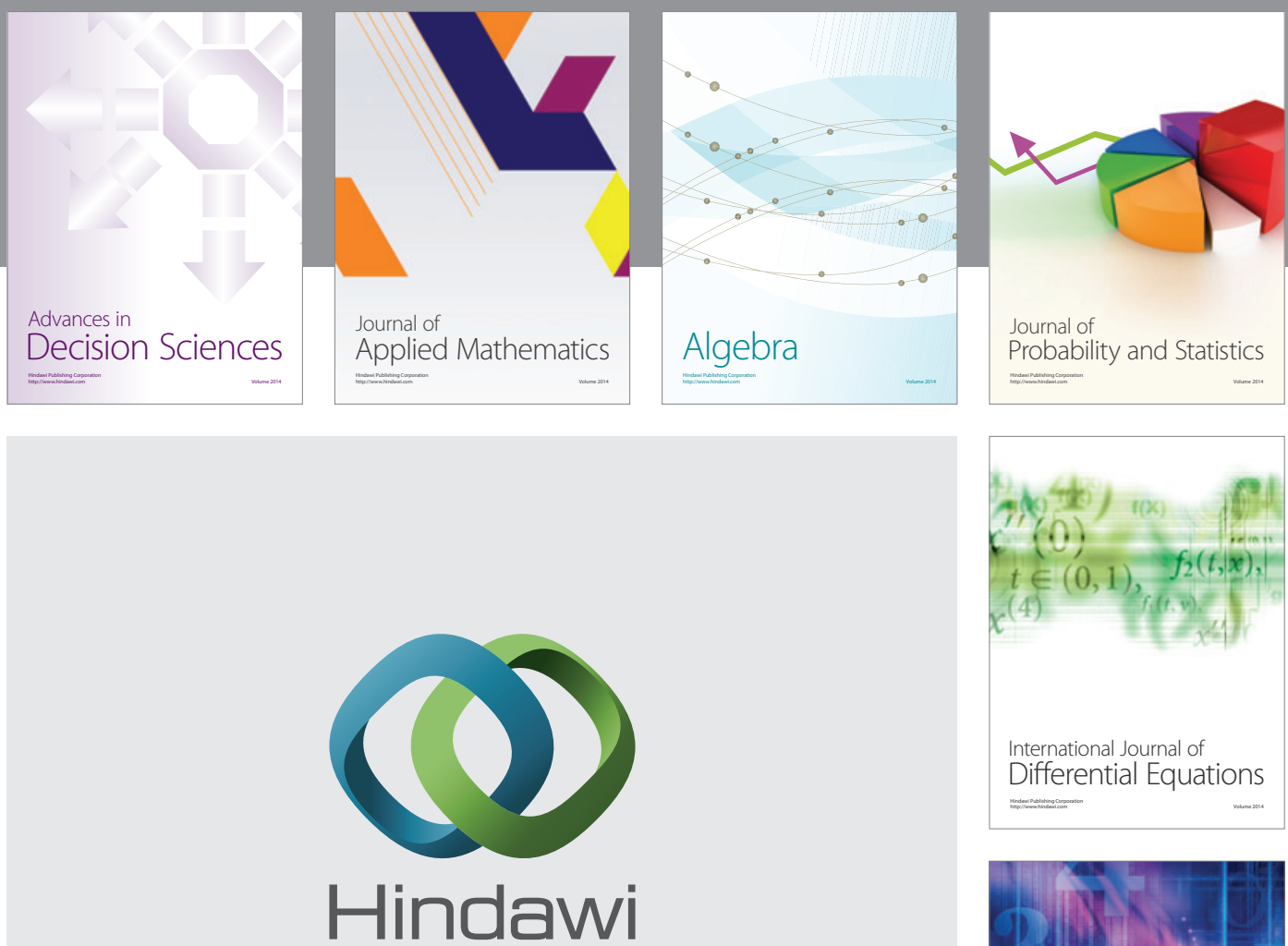

Submit your manuscripts at http://www.hindawi.com
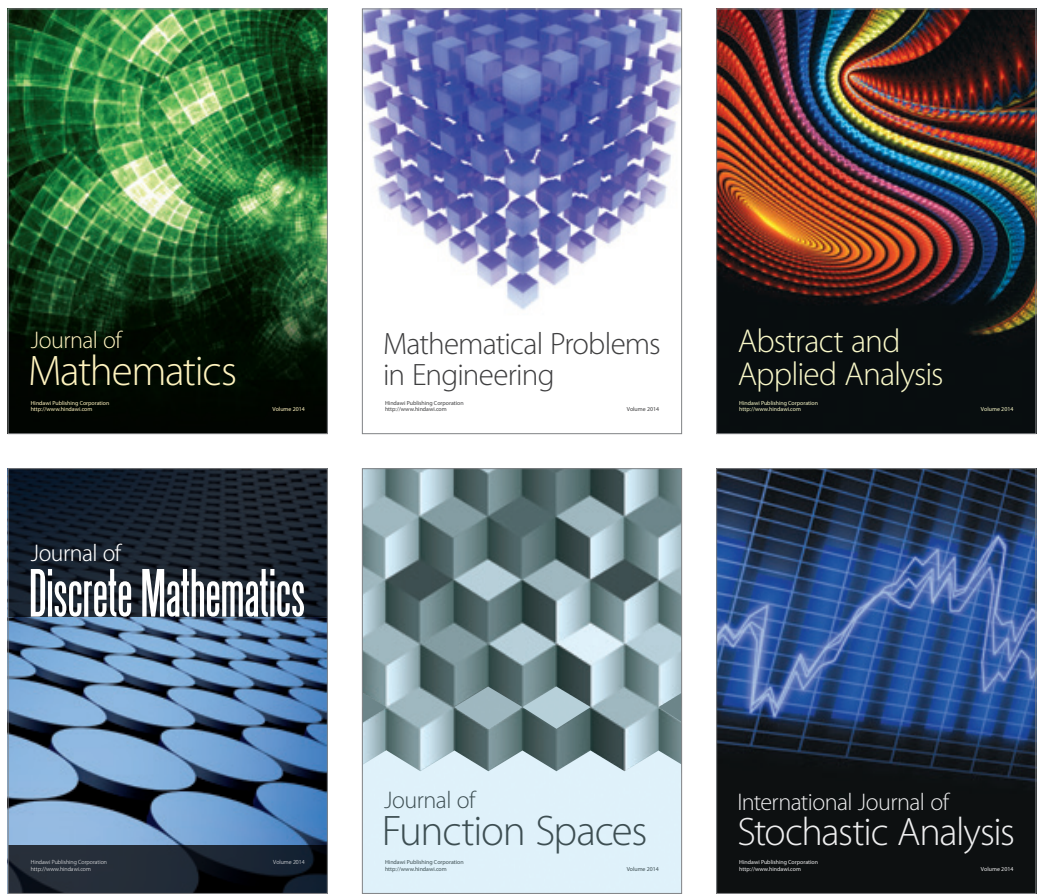

Journal of

Function Spaces

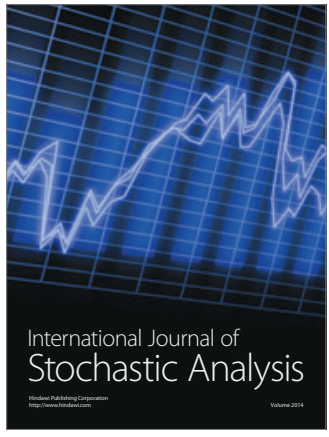

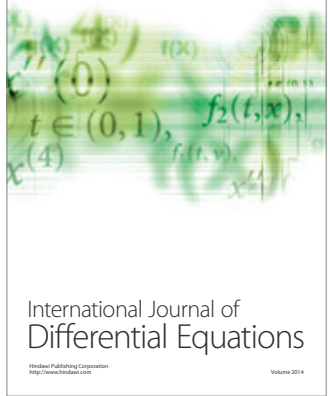
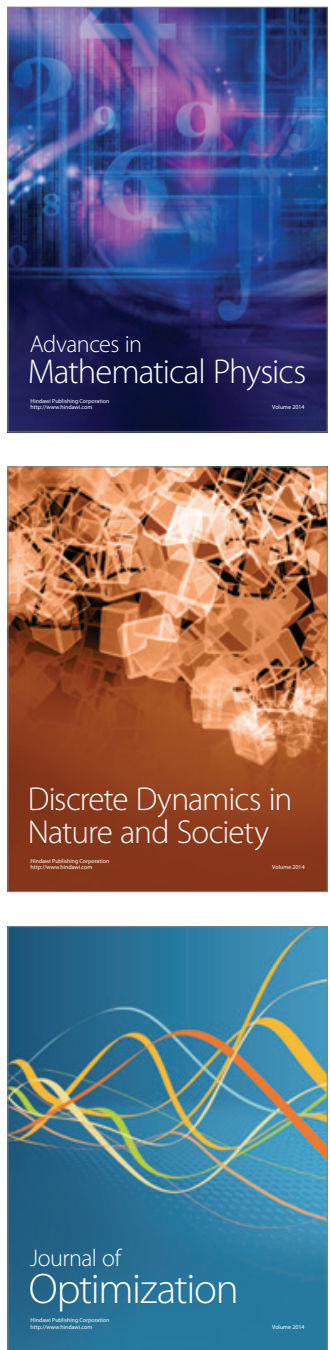